\title{
Las guías de estudio en la Universidad Estatal a Distancia: de lo remedial al dispositivo pedagógico
}

\section{Dagoberto Núñez Picado}

Investigador, CICDE, Universidad Estatal a Distancia; dnunez@uned.ac.cr

Recibido: 6 de abril de 2010

\section{RESUMEN}

Este artículo se aboca a determinar las características fundamentales de las guías de estudio como instrumentos idóneos para el comentario de textosexpertos $y$, de modo especial, para alfabetizar sobre la naturaleza de las ciencias, en tanto forma particular de conocimiento y fuente del currículum, para diversas asignaturas, en distintas carreras de la Universidad Estatal a Distancia (UNED). De modo particular, se subraya que la guía de estudio se torna una necesidad toda vez que crecientemente las cátedras recurren a textos de mercado cuyos enfoques y contenidos requieren de una mediación pedagógica correspondiente con la realidad nacional.

\section{PALABRAS CLAVE}

Transposición didáctica, discurso reproductivo, comentario de texto, alfabetización sobre la naturaleza de las ciencias, mediación pedagógica, texto-tutor y texto experto.
Aceptado: 16 de setiembre de 2010

\section{KEY WORDS}

Didactic transposition, reproductive discourse, comment text, conception of the Nature of Science, pedagogical mediation, mentor text and expert-text.

\section{INTRODUCCIÓN}

En la Universidad Estatal a Distancia (UNED) de Costa Rica, cuando se tiene un texto de mercado para un curso, se plantea la producción de una guía de estudio que adapte dicho texto a las características del sistema de educación a distancia. La historia de la producción de materiales didácticos de la UNED muestra una variopinta gama de modalidades de producción de guías de estudio. Como consecuencia no tememos arriesgar propuestas de producción de guías de estudio, con base en los resultados de la investigación "Guías de estudio para libros de mercado" realizada durante el año 2009. Una valoración crítica general del tema permite afirmar que lo realizado 
en el pasado, por una parte, puede llevarnos a consagrar el trabajo de producción académica reduciéndolo -en el presente- a un frío esquema forjado desde la complaciente comodidad de unas secciones fijas, jalonadas más por la fuerza de la costumbre que por aquello que proviene de una evaluación académica fundamentada y sustantiva.

Por otra parte, de modo propositivo y no siendo la actitud señalada la común, existen dos funciones históricas de la guías de estudio en la UNED de Costa Rica, que pueden seguir aportando, considerada su evolución institucional, positivos dividendos: la producción de guías de estudio ha representado -a lo largo de tres décadas- un instrumento cuya finalidad apunta a alfabetizar sobre la naturaleza de las ciencias y, junto con esta función, por otro lado, vemos que dicha producción se ha caracterizado, discursivamente, por echar mano de un tipo predominante de estilo didáctico -identificable-bajo el término clásico de "comentario de texto".

Ambas funciones nos parecen de sumo valor; porque las guías de estudio consiguen por sí mismas cierta legitimidad en la UNED; la alfabetización sobre la naturaleza de las ciencias y el recurso al estilo propio de los clásicos comentarios de texto, dan carta de ciudadanía a las guías de estudio, con una larga trayectoria académica. Asimismo, consideramos que nada impide que ambas funciones sean sustentadas bajo cierto marco teórico que permita que los mencionados instrumentos didácticos sean académicamente evaluados en sus alcances teóricos y prácticos. ${ }^{1}$

Por lo anterior, este artículo delimita su objeto de estudio en dos planos, por una parte, se separa de la idea que justifica un solo patrón global de producción de materiales didácticos para la UNED-Costa Rica y, a la vez, propone la idea de una producción histórica de guías de estudio para la UNED, que proporcione bases críticas, en todo lo que este tipo de instrumento pueda aportarle al proceso educativo para su debido desarrollo; el hecho de que la UNED recurra a textos de mercado como fuente principal de conocimiento científico para la administración de sus cursos, se visualiza así en una oportunidad para la renovación que exige la realidad social costarricense.

\section{- Problematización respecto de la producción de guías de estudio en la UNED}

A propósito de los elementos y relaciones estructurales de las guías de estudio, este artículo provee un sistema conceptual de análisis para las actividades prácticas de diseño, construcción y evaluación de dichas guías. En ese sentido, se inscribe -académicamente- como una contribución a la investigación de la administración académico-educativa que realiza la UNED.

De acuerdo con la necesidad de desarrollar el sentido de las principales funciones que adoptan las guías de estudio utilizadas en la UNED, requerimos de la exposición del contexto general sobre su origen, así como explicitar los principios epistemológicos que, como universidad pública, acuerpan su elaboración, aunque sea introductoriamente. La serie de aspectos señalados de seguido, tienen la finalidad de aportar de modo resumido estos aspectos.

» Existe en el mercado del libro muy diversas opciones de materiales impresos a los cuales puede acudir la UNED: algunos de ellos no tienen ninguna pretensión científica, por consiguiente sería absurdo esperar de ellos tal aporte. Consideramos que el conocimiento científico es un tipo de conocimiento y no el único y ni siquiera el más importante. Pero, cuando se trata de seleccionar un texto de mercado o experto (entendiendo por tal cualquier fuente para el nivel superior de educación) con función pedagógica -a propósito de que sirva para elaborar una guía didáctica-casi sobra señalar que tal material, 
debe resultar guiable; si el texto seleccionado fuera insuficiente o totalmente deficitario en cuanto a la pertinencia o calidad de sus contenidos, la guía de estudio tendría que asumir la tarea de comunicar esos contenidos ya que el texto-experto no lo logra, esto llevaría a preguntar -justamente- ¿para qué ese texto-experto?

Una forma de asegurar una selección pertinente de contenidos podría ser -según las orientaciones del diseño curricular- producir una antología o compendio de artículos con resultados de investigación, desde donde se pueda partir para producir, con todo el sentido del término, la guía de estudio; los materiales así compilados pueden satisfacer directamente la necesidad de los contenidos para una asignatura determinada.

La UNED -como entidad de educación superior-adquirió un compromiso ético en torno al conocimiento científico, fundamento de la formación profesional que ofrece: mucho de nuestra legislación (vía congresos internos, por ejemplo) lo apuntala, en términos de ofrecer un aporte socio-cultural y económico al desarrollo del país, por ello bajo ningún concepto la UNED puede ignorar o renunciar a esta obligación ${ }^{2}$. En consecuencia, la producción de guías de estudio, no puede plantearse a contrapelo de ese compromiso científico. El eje transversal de investigación procura ser consistente con el planteamiento.

Desde el punto de vista estadístico - globalmente-se debe tener claro que aunque en los últimos años ha crecido la demanda de guías de estudio, ésta no ha superado en número a las unidades didácticas, en las tres décadas 3 . Lo anterior obedece -según los datos- a que existen más claros lineamientos de las funciones de una unidad didáctica (incluyendo la unidad didáctica modular) que los referidos a las funciones propias de las guías de estudio. Y desde el punto de vista de la producción académica -tal y como se realiza en la UNED - las guías implican un trabajo de producción de mayor complejidad técnica, en comparación con la producción de otros medios didácticos como lo son las unidades didácticas u otros dispositivos didácticos que no requieren el esfuerzo de ser re-pensados a partir de otro texto.

Una razón de valor epistemológico que subyace a la programación curricular de guías de estudio estriba en analizar su utilidad, desde el punto de vista de su diseño, para aquellos cursos que en la UNED sirven para varias carreras ${ }^{4}$. La situación obligaría a repensar diseños de guías cuyos rasgos, en su mediación pedagógica, se avengan con esas necesidades particulares. En el fondo, y por ahora, los cursos de servicio exigirían una flexibilidad intrínseca de las guías de estudio en tanto herramienta con una vocación de larga y mejor vida útil en la UNED. Sin olvidar, por supuesto, la siempre en relativa autonomía connatural de la guía de estudio respecto del libro de mercado, puesto que esta condición de origen la constituye -necesariamente- en una obra derivada de dicho libro.

»Entre los prolegómenos que venimos esbozando para contextualizar el sentido de las guías de estudio de la UNED no debe soslayarse que su finalidad principal estriba en canalizar los objetivos de un curso dentro de la curricula de la UNED. En otras palabras, la UNED no produce guías de estudio para repetir o comentar un libro de mercado, de por sí. Lo hace en función del cumplimiento de unos objetivos y de unos contenidos programáticos que están 
canalizando un plan de estudios dentro de su sistema de educación a distancia.

En la UNED, durante la década que agoniza, se ha producido una creciente demanda de guías de estudio que refleja tres aspectos correlacionados entre sí. Bajo esos aspectos podemos reconocer cierta dinámica y perfil educativo institucional, por eso se requiere analizarlos por separado.

\section{a) La demanda de guías de estudio para} libros de mercado (GEPLM) como recurso didáctico: su demanda creciente se origina a raíz del acceso a libros de mercado que cumplen con los contenidos de los cursos que imparten las cátedras. Cuantitativamente, podemos afirmar, se trata de una demanda desigual; las escuelas no demandan las guías de estudio en la misma cantidad o para satisfacer iguales necesidades pedagógicas. También, desde el punto de vista cuantitativo, la escuela que lidera cuantitativamente la demanda de guías de estudio, es la Escuela de Administración ${ }^{5}$ y la razón que más destaca para esta solicitud tiene relación, de fondo, con el carácter cambiante de las Ciencias Administrativas -tal como se practica en la UNED- así como por un enfoque administrativo vinculado estrechamente a los planteamientos internacionales de administración de la sociedad norteamericana (EE.UU.).

Según la interpretación del Director de la Escuela de Administración "transitamos por momentos en que la coyuntura internacional genera, constantemente, modelos de gestión liderados por autores y escuelas de pensamiento en el campo de la Administración"6 y, la política académica consiste en permanecer "atentos" a esas corrientes de cambio para corresponder curricularmente con respuestas académicas que "aprovechen" las situaciones y lineamientos norteamericanos, en "beneficio" de la realidad nacional y nuestros estudiantes.

b) La instrucción institucional: otras cátedras de las distintas escuelas de la UNED, acuden a las guías de estudio para remediar una cuestión práctica: la Vicerrectoría Académica dictaminó que ninguna cátedra debería ofrecer un texto de mercado, para satisfacer la demanda de un curso, sin la debida producción de la guía de estudio respectiva y se acató la llamadar. Este mandato marcó también el terreno para que estadísticamente aumentara -durante la última década - la demanda de este dispositivo pedagógico, por lo demás, originado con el nacimiento mismo de la UNED.

Conceptualmente, conviene señalar que los materiales didácticos que suelen venderse en el mercado del libro están diseñados dentro del marco de la educación presencial por lo cual su aplicación en la UNED puede resultar problemática; los libros de mercado, en términos educativos, suelen ser libros no didácticos; aquí los denominamos texto-experto distinguiendo que son producidos -según Chevallard- como fuentes de un "saber sabio" (en términos metafóricos ya que evidentemente se trata de una producción textual también transpuesta). Las guías de estudio en relación con el texto experto, configuran más una obra derivada que reproduce (comentando) aquel saber del texto-experto.

c) La UNED y su autonomía universitaria: bajo un espíritu crítico y de prevención -desde el punto de vista académico- el fenómeno de la producción de guías de estudio para libros de mercado, generó la duda sobre si la UNED estaría comprometiendo su independencia en materia de contenidos curriculares, al 
amarrar su trabajo académico a un libro de mercado del cual sólo hace una guía de estudio; ¿arriesgará la UNED de esta manera su libertad de cátedra? Si se observa que este trabajo de transposición -representado en la guía de estudio- está regulado por una descripción curricular que -a su vez-depende de un plan de estudios, entonces se nota que la UNED no estaría sujetando los contenidos curriculares de modo azaroso; esos contenidos extraídos del textoexperto, estarían conformes con una planificación curricular; no se trata de una apuesta -irreflexiva-por los contenidos mismos del libro de mercado per se; esto sí nos dejaría en una condición académica dependiente.

Sí preocuparía que en alguna situación ambivalente la administración de los cursos llevara los contenidos curriculares a merced de propuestas de contenidos de libros de mercado de calidad discutible ${ }^{8}$ y sin los controles de calidad respectivos. Además, en dicho caso, esos contenidos curriculares presentes en los libros de mercado podrían replicar ciegamente una visión cultural que omite los principios de autonomía académica que rigen a una universidad pública.

De modo realista, es predictible también otra amenaza, nos referimos a la económica, tan evidente en la coyuntura recesiva internacional por la que pasamos; y es que la elevación de los costos de transporte y de almacenamiento de los libros de mercado puede disparar los precios de los libros, convirtiéndolos en una mercancía aún más cara y desde ese punto de vista inalcanzable para los regateados fondos públicos que nos gira el Estado.

El régimen de costos atentaría en contra de la sostenibilidad fundamental de todo el sistema educativo de la UNED: como centro de educación superior con una modalidad en la cual los textos escritos siguen siendo el pivote sobre el cual se soporta como sistema, la amenaza sería grave.

d) ¿Cómo seleccionar textos-expertos "guiables"?: Toda la problematización anterior conduce a que las Escuelas de la UNED consideren la guía de estudio como una herramienta didáctica oportuna, que habría que aprovechar, sobre todo ante la declarada y reconocida inopia de autores para hacer unidades didácticas, o incluso para renovar las que requieren actualización.

Esta posición - por lo menos, legal-coincide con la tesis de que las guías de estudio en la UNED representan un dispositivo didáctico mínimo que ayuda a regular curricularmente el estudio de los libros de mercado. Esa relación sugirió nuevas dudas ante un doble peligro académico: aún cuando se realizara un buen estudio para la estructura de precios, persistía el peligro sobre la calidad en la selección de textos de mercado en atención a los conocimientos curricularmente requeridos.

Se consensuó que una inadecuada selección impide, o al menos hace más trabajosa, cualquier empresa de producción académica posterior. Para la situación señalada no faltaron discusiones que apelan a la sana preocupación respecto de una excelente selección de libros de mercado, mediante el reconocimiento del lugar central que ocupan las personas especialistas en el campo de conocimiento respectivo, antes $y$ durante la elaboración de las guías de estudio; es decir, tanto en la selección como en la producción.

Si nos preguntamos por las opciones teóricas que tenemos como potenciales fuentes para la producción de GEPLM, se configura cierto 
esquema que idealmente representa -de forma figurada- una flecha de dos puntas con infinidad de formas intermedias:

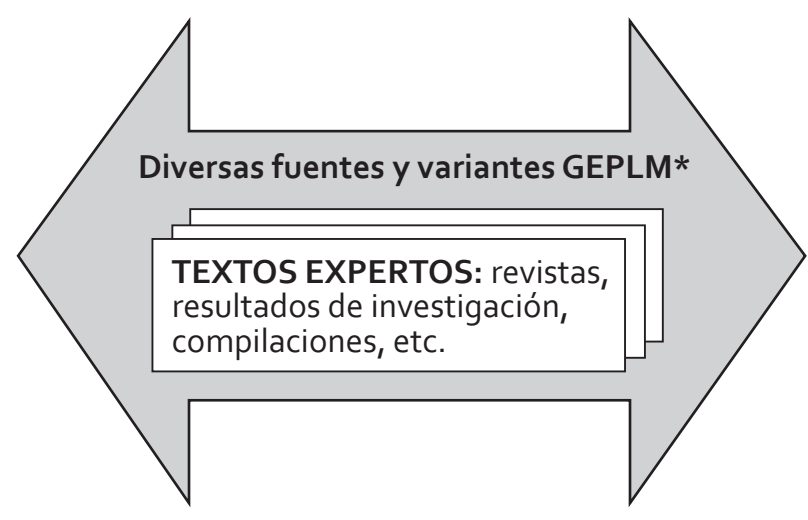

En un extremo estaría la opción de asumir materiales externos (con derechos ajenos de autoría, en otros idiomas distintos del español, etc.) incluyendo aquellos convertidos - por encargo a editoriales externas -en textos didácticos para la UNED9. Ese primer lado (supongamos que es la punta derecha de la flecha) representaría un tipo de fuente curricular para la UNED, que nos dice que para producir guías de estudio, prácticamente no existen límites en cuanto recurrir a fuentes externas; en el otro extremo encontraríamos -tendencialmente- el grave problema de una selección inadecuada que haría inviable producir guías de estudio pertinentes, sea en razón de la baja calidad de esas fuentes o porque las unidades de conocimiento de esos textos expertos no son las que corresponden curricularmente.

$Y$, en el centro del esquema, encontraríamos la típica guía de estudio (bajo soporte impreso o digital) que responde -efectivamente- a la necesidad de transponer contenidos para un curso particular: las fuentes también son infinitas. Prácticamente, no existe fuente de conocimiento que no sea factible de encontrar su mediación pedagógica vía guías de estudio, para un curso determinado.
Notas para un marco metodológico de producción de guías de estudio

El diseño de las guías de estudio puede ser definido como una acción didáctica-educativa-de traducción (Echeverría, 2004:10 ${ }^{10}$ ) de un cuerpo de conocimientos ya estructurado (texto de mercado) a otro que hay que estructurar (guía de estudio). Esta labor, dentro del sistema de educación a distancia de la UNED, entraña funciones ineludibles.

Una visión general de esta labor particular de transposición didáctica, desde el punto de vista de su mediación pedagógica, es entendida aquí desde dos aspectos que le son inherentes: por una parte, en cuanto responde a una necesidad que le da su origen como dispositivo pedagógico (¿para qué nació esa herramienta didáctica?) y, por otra parte, en tanto designa ciertos elementos discursivos constitutivos desde los que construye su aporte en el plano de la acción educativa en la que se inscribe.

\section{La Guía de estudio realiza la función vali- dadora de la naturaleza científica del tex- to-experto:}

El sistema de educación a distancia aplicado en la UNED permite separar -formalmentelos procesos de enseñanza de los procesos de aprendizaje. Cada uno de esos territorios (el de la enseñanza y el del aprendizaje) supone dos tipos de validaciones, según los destinatarios de ellas: la validación de enseñanza (o de contenido según el tipo de conocimiento) permite situar la naturaleza científica de un texto experto y responde -en principio- a una tarea de evaluación de la calidad académica de una fuente, en cuanto a la validez de sus planteamientos; su posición epistemológica, los criterios de verdad y los conceptos fundamentales. Lo que corresponde a este ámbito de validación solo nos lo pueden corroborar expertos en áreas de conocimiento particular, sería absurdo someter a los estudiantes la validación en este plano; sería como colocar la tarea de la enseñanza de 
una institución de educación superior, en el nivel del gusto o disgusto de un estudiante ${ }^{11}$.

El discurso científico se toma aquí como sinónimo de discurso producido desde un(os) campo(s) de conocimiento, por expertos (as). $Y$ si esos conocimientos -consignados en un texto de mercado- requieren de una guía de estudio, su proceso de análisis y de producción estará condicionado en su estructura básica por aquel. Pero, además, se plantea -inversamente- que una buena selección de un libro de mercado depende de su potencialidad para inducir ciertos rasgos propios a la estructura y funcionamiento de la guía de estudio correspondiente, en el contexto del curriculum de una carrera.

\section{Función de comentario del texto-tutor:}

La Guía de estudio, por consiguiente, es una obra derivada que permite validar (comentar, criticar, resumir, etc.) la obra fuente o texto experto, en vista de su actualidad científica, del manejo conceptual, del estado del arte, de la pertinencia y cohesión epistemológica y de su coherencia gnoseológica. La naturaleza didáctica de una guía de estudio hecha por la UNED es parte de un discurso reproductivo inspirado en el discurso productivo de la obra fuente: en ese sentido el discurso del tipo comentario de texto (las características pedagógicas del lenguaje de este discurso reproductivo) es connatural a la producción de la guía de estudio; esto significa que para construir la mediación pedagógica de la guía de estudio -su transposición didáctica en relación con el texto experto- no repugna para nada que se acuda a la forma y contenido de un comentario, en el sentido clásico de la tradición académica y humanista; lo que se tiende a hacer es comentar fragmentos, capítulos, palabras, conceptos, etcétera, del texto-experto.

Como corolario de lo señalado vemos dos puntos que se destacan: Primero, se deben tener presentes dos principios decisivos en materia de producción de textos derivados, como es el caso de las guías de estudio: ellas, por una parte, no dicen -sustantivamente- nada formalmente contradictorio con los textos-expertos; sí, en complemento o en línea crítica respecto de lo que el texto-experto expresa. $Y$ podemos concluir que, en todo caso, una guía de estudio dialoga, desde el área disciplinaria o interdisciplinaria propia, con el texto experto y es desde ahí que aporta los aspectos críticos, complementarios, etcétera.

Un segundo punto estriba en señalar que los objetivos de aprendizaje delimitados en las descripciones curriculares influyen directamente (direccionan) la producción de la guía de estudio. En general el propósito general que gobierna esos objetivos de aprendizaje delimitará la selección del texto de mercado.

En conclusión, conjuntando las dos funciones esenciales de las guías de estudio (alfabetizadoras de conocimientos y comentadoras de textos) vemos que cualesquiera sean las propuestas de formatos de guías de estudio, ellas conducen a proveer sistemas conceptuales de análisis planificados curricularmente, respecto de los libros de mercado.

\section{- Lineamiento epistemológico para la producción de guías de estudio en la UNED-Costa Rica}

La doble función sustantiva de las guías de estudio (finalidad de alfabetización sobre la naturaleza de las ciencias mediante el comentario de textos-tutores) diseña ciertas disposiciones prácticas a la hora de ser producidas por los equipos de trabajo.

En ese sentido, el análisis de textos-expertos potables reside en discernir si sus unidades de conocimiento llenan -satisfactoriamenteciertas necesidades curriculares. Se trata de una labor orientada por los planes de estudio. En esa dirección, la pregunta inicial es: ¿Qué es lo "generatriz" de la guía de estudio? 
La estructura virtual de la guía de estudio no es sin más expresión de la estructura efectiva (fundamento epistemológico-conceptual) del libro de mercado, lo es en el marco de un curso o asignatura y dentro del plan de estudios correspondiente: por la complejidad de este contexto, valga señalar que este momento "generatriz" o generador, es del todo desaconsejable conceptuarlo como un trabajo en solitario, por parte del encargado de cátedra o de programa, del productor académico o algún especialista, sin los auxilios básicos de una descripción curricular cuyos objetivos de aprendizaje estén claramente definidos y cuyas metodologías estén bien diseñadas.

Este enfoque nos liberaría de cierto patrón didáctico único, ya que no cabe esperar de un equipo de trabajo la simple reproducción de un esquema único que ha prevalecido por más de tres décadas en la UNED, y que se le impone externamente a todos los contenidos curriculares, desde una supuesta experticia didáctica: si nuestras guías de estudio deben presentar algunas secciones fijas correspondientes a los objetivos, resúmenes, ejercicios de autoevaluación, etcétera, para ello vale destacar cuáles serían las razones fuertes que acreditan tal patrón; ¿se desprenderá del carácter disciplinario propio del conocimiento que se trate? En esa dirección cabe detenernos en varios aspectos de esta interrogación:

a) Las diversas ramas de las ciencias divergen no solo en su contenido, sino también en su funcionamiento y concepción cognoscitiva. Estas disimilitudes resultan particularmente claras en las ciencias humanas y en las naturales, muy especialmente en cuanto al tipo de discurso que las caracteriza. Mientras el paradigma de las ciencias naturales busca construir una imagen de la realidad que pueda contrastarse y comprobarse en relación con ella misma, el discurso de las ciencias humanas es interpretativo, desde el momento en que se pregunta por el significado del ser humano (mujeres y varones) y del universo.

b) Por consiguiente, al seleccionar un texto-experto la valoración (de sus temáticas) debe partir de una gran diversidad de contenidos, funcionamientos y concepciones cognoscitivas, según la rama de las ciencias de que trate: Por ejemplo, en un texto de ciencias naturales se valorarán sus significados (verificables), tendencialmente correlacionados unívocamente entre sí. En cambio, si se trata de un texto que pertenece a las ciencias humanas su valoración partirá de criterios emanados de la naturaleza del lenguaje, particularmente dos: el de coherencia interna y externa del discurso (capacidad para buscar nuevas unidades que combinen las existentes con las presentes en otros discursos) y el de riqueza de significados (explotación de la polisemia tipificadora del lenguaje $)^{12}$.

En segundo lugar, al comentar el textoexperto, toda guía de estudio realiza una descripción y una evaluación del mismo, labor de suyo muy compleja en vista de las presuposiciones que comporta. Sin embargo, estas tareas propias de producción de una guía (tarea de descripción y de evaluación de un texto-experto) significan resumidamente lo siguiente: Por lo que toca a la descripción, se trata de la interrogación sobre lo que hay en el texto-experto y cómo se presenta. Por lo que respecta a la evaluación del texto-experto, se cifra en la pregunta relacionada con los méritos o deméritos que posee dicho texto para efectos de enseñanza/aprendizaje dentro de un sistema de educación a distancia.

En tercer lugar al comentar el libro de mercado toda guía de estudio deberá ser discursivamente alineada al discurso reproductivo que le es propio; esa función del trabajo educativo va 
modelando para efectos de estudio como parte del proceso de adquisición del lenguaje- cierta estrategia a propósito del tipo de conocimiento para el cual se entrena el alumnado.

c) La determinación del enfoque teóricometodológico del quehacer educativo resulta indispensable para la debida transposición didáctica que está implicada en la producción de guías de estudio. Este punto debe ser señalado a modo de un gran ejemplo -especialmente por lo que atañe epistemológicamente al asunto- y tomamos para ello la tradición filosófica en Francia que acoge adquisiciones de ciencias de la educación y de la psicología del aprendizaje -especialmente de la corriente constructivista representada por Piaget y Vygostsky (Tozzi et ál., 1992), y se inclina -notoriamente- por el derecho de que la filosofía llegue a todos los alumnos, sin excepción. La premisa de esta campaña es que un contacto -al menos propedéutico- con el saber filosófico, trae beneficios académicos y culturales al estudiante que de faltarles no podrían llenar -tan directamentepor otros medios. Quienes impulsaron este cambio en la educación filosófica, formularon sus tesis, y podemos ayudarnos con ellas para ilustrar el tipo de problema intrínseco a un tratamiento didáctico responsable.

Entre los arquetipos de la enseñanza filosófca (partiendo de Galichet, 2000 y Toszi, 1992) cabe distinguir varios paradigmas organizadores del discurso filosófico susceptibles para estructurar una enseñanza de la filosofía fiel, por ejemplo, al modelo de comentario de texto, que hemos aludido como típico de las guías de estudio de la UNED. Los autores sistematizan cinco arquetipos de enfoque educativo en educación:

" Arquetipo histórico: este enfoque se sustenta en lo que tradicionalmente se denomina historia de las ideas, situándolas en el contexto pasado o presente. Este sería el caso de los países latinos.

» Arquetipo doctrinal: esta perspectiva es característica de la enseñanza de la filosofía dentro de marcos "oficiales"; es el caso -extremo- de la enseñanza de Marx en países comunistas o del paradigma tomista en la España franquista.

»Arquetipo positivista: este enfoque se apoya esencialmente en las ciencias humanas, en la adquisición de reglas de lógica formal, los datos de la epistemología y de la historia de las ciencias.

» Arquetipo praxiológico: La propuesta pragmatista reflexiona a partir de las experiencias de diálogo de los estudiantes, alimentando la formación ética, perfilando métodos para abordar dilemas morales (en línea con la propuesta técnica de Kohlberg, actualmente desarrollada en Bélgica y Canadá).

" Arquetipo problematizador: Este enfoque tiende a extraer los problemas a partir de nociones y a desarrollar en el alumno un cuestionamiento más allá de dar respuestas. Se acerca -según Galichet- a los objetivos de filosofía para niños de Lipman. Esta corriente didáctica reúne la problematización junto con el aprendizaje de competencias, la puesta en situación, y la búsqueda del desarrollo personal y grupal.

El derecho a la filosofía para todos se nos presenta y justifica aquí, a guisa de ejemplo, como desafío e invitación para proponer bases sólidas a nuestras propuestas didácticas, según cierto enfoque curricular. Ejemplificando con el caso de un curso de Administración Financiera, se estaría postulando la importancia de metodologías y objetivos de predicción de las consecuencias de los manejos macro-económicos 
que pudieran analizar la generación de crisis financieras, como las originadas por problemas hipotecarios, de tal manera que quienes ejerzan la profesión no ignoren que algunos manejos de cierta lógica financiera, producen algunos resultados catastróficos. Sobre todo porque -parafraseando- las cosas de la lógica son una cosa, mientras que la lógica de las cosas podría ser totalmente otra.

\section{- Recomendaciones y pistas de producción de las guías de estudio}

Para la apasionante tarea de asesoramiento de producción de las guías de estudio, emergen una serie de pistas y recomendaciones que se desprenden de lo expuesto; a saber:

a) Identificamos una primera condición interna de lo didáctico -en el contexto UNED- que remite al discurso propio de las disciplinas científicas particulares. Esto significa que el discurso didáctico pasa por el enfoque o los enfoques de dichas disciplinas antes de recibir los tratamientos educativos, propiamente dichos. Consecuentemente el acto-educativo vía guías de estudio, en el trabajo didáctico de la UNED, implica un posesionamiento claro de el o los enfoques epistemológicos que buscará promover desde las áreas disciplinarias o multidisciplinarias correspondientes.

b) Una segunda condición interna de lo didáctico -en el contexto UNED- remite a los planes y programas diseñados desde ciertos enfoques curriculares: en la UNED hay que investigar las características de estructura y contenido de los planes y programas para determinar las modalidades educativas que configuran y que subyacen a los procesos formativos de las distintas carreras.

c) El discurso reproductivo en las guías de estudio depende -directamente- del discurso producido en los textosexpertos, por consiguiente la disciplina plasma la estrategia modeladora; esto es, la función formativa, científica y profesional que la UNED realiza con sus estudiantes. En ese sentido, el discurso reproductivo (por ejemplo, el del tipo comentario de texto-experto) contiene una raíz meta-cognitiva que le es esencial y que es aplicado para explorar, como propiedad del trabajo didáctico, algunas estrategias de enseñanza según algunos estilos de aprendizajes pre-determinados.

d) No hay que perder de vista que tanto el discurso productivo como el reproductivo modelan a su manera: sin embargo, es el discurso reproductivo el que ofrece la oportunidad de comprobar si el estudiante está comprendiendo una materia porque se ajusta a sus estilos de aprendizaje o si -por el contrario- habría que variar la estrategia potenciadora de autorregulación de los aprendizajes.

e) Al explicitar los principios y lineamientos productivos del recurso educativo del tipo guías de estudio, asistimos - no por casualidad-a la función esencial de la tarea didáctica dentro del sistema de educación a distancia de la UNED. En el informe final de la investigación Lineamientos y criterios para la producción de Guías de estudio para libros de mercado se presentan, a modo de principios y lineamientos, una amplia propuesta de instrumentos didácticos atinentes al diseño y el análisis de este dispositivo pedagógico bajo la teoría de Transposición Didáctica (Chevallard, 2000) y de la metodología de Regulación y Autorregulación de Aprendizajes (Jorba y Casellas, 1997).

f) La vía de cotejar y analizar en qué sentido las guías de estudio hacen la tarea propia del discurso reproductivo 
(comentario) que les corresponde -partiendo del discurso productivo del libro de mercado- es una vía directa y crítica de investigación que trasciende la visión de la guía como simple recurso educativo remedial y la lleva al rango de dispositivo de estudio de las condiciones y la fundamentación inherente de lo didáctico dentro de la modalidad educativa de la UNED, bajo determinados parámetros epistemológicos propios.

g) No contradice el concepto de guía de estudio de la UNED-Costa Rica, ni tampoco su práctica histórica, el que dicho recurso educativo se construya como una obra derivada que reúna varias fuentes de conocimiento científico (tipo antología, compilación de artículos o de resultados de investigación, etcétera) y tampoco lo contradice $-y$ hay evidencias en validación- de su materialización en soporte digital.

h) En el caso de la enseñanza de las ciencias de la administración vemos muy importante la construcción de propuestas, por parte de especialistas en el tema, de enfoques teórico-epistemológicos en función de la enseñanza del mismo tipo que ejemplificamos como arquetípicos en filosofía. Los paradigmas organizadores del discurso en las Ciencias de la Administración harían un servicio epistemológico de primer orden para la producción de guías de estudio que sean capaces de describir y evaluar, a fondo, los textos de mercado por utilizar en las diversas asignaturas, dentro de las carreras.

El tipo de discurso reproductivo (acompañante y aprendiz) al que pertenece la guía de estudio, como discurso secundario, contiene el ideal de "hacer cantar aquella ciencia" a la que remite, de la que depende y a la que le sirve como vehículo desde el cual la población estudiantil puede aprender a manejar los conceptos básicos dentro del campo de conocimiento en que aprende a moverse.

\section{NOTAS}

1. En este artículo postulamos una línea de producción de guías de estudio que implemente aquellos considerandos esbozados en el Modelo Pedagógico y en los Lineamientos de Política Institucional (2007-2011), de la UNED. Ver: UNED. (2009) Directrices de la UNED. ClDREB. San José, Costa Rica.

2. El 4 de julio de 2003 el Consejo Universitario aprueba que el problema académico "Investigación" constituye el quinto eje transversal de la Universidad Estatal a Distancia (acta 1656-2003). En línea con ese compromiso académico la UNED inauguró su trabajo del año 2009 con la visita del epistemólogo Dr. Dennis Paul Huffman Schwocho de la Universidad Autónoma de Chapingo, quien impartió el Seminario-taller Métodos y metódica científica; en él trató temáticas ligadas a la naturaleza propia del quehacer científico. Programa de Fundamentos de Investigación en Educación a Distancia (PROIFED). Vicerrectoría de Investigación. UNED.

3. Ver: Grafico 1 de Núñez $(2009,23)$ Lineamientos y criterios sobre Guías de estudio para libros de mercado, en UNED-Costa Rica. Informe Final de Investigación. Vicerrectoría de Investigación. UNED. En el gráfico se consigna que es a partir del año 2005 al 2008 que se comienza a observar una creciente demanda de guías de estudio.

4. De hecho este elemento es importante para la decisión de producción de guías de estudio y está inspirado en una evaluación, por parte de un equipo de investigadoras (Zúñiga, et ál.) que plantea la necesidad de una o varias guías de estudio para cursos de servicio. Ver el texto: Claramount M.C. (2006). Casitas quebradas: El problema de la violencia doméstica en Costa Rica. Décima reimpresión. EUNED. San José, Costa Rica. La investigación se titula: Estudio evaluativo y sistematización de la experiencia educativa del curso, Elementos Generales acerca de la Violencia doméstica. CIA. Vicerrectoría Académica. UNED. Zúñiga et ál. (2001, Octubre) Estudio evaluativo y Sistematización de la Experiencia Educativa del Curso: Elementos Generales acerca de la Violencia Doméstica: Propuesta de reestructuración. Centro de Investigación Académica. Vicerrectoría Académica. UNED. $106 \mathrm{p}$.

5. Ver: Cuadro 3 de Núñez $(2009,22)$ Lineamientos y criterios sobre guías de estudio para libros de mercado, en UNED-Costa Rica. Informe Final de Investigación. Vicerrectoría de Investigación. UNED. En el cuadro se consigna 
que del año 2005 al 2008 ECA-UNED solicita 52 guías de estudio, seguida por la ECSH con 33 solicitudes.

6. En opinión del Director de la Escuela de Administración (entrevista del 4 de Mayo, 2009), tal coyuntura resulta coincidente con la línea de formación de su Escuela en la UNED. Señala el Dr. Gutiérrez que "la UNED puede conocer desde adentro" la materia administrativa con nociones originadas en U.S.A. Incluso, esta Escuela ha convenido con una importante editorial con el fin de que libros - propiedad de dicha editorial - reciban la traducción al castellano con ejemplificaciones ofrecidas para el estudio de casos propios de Costa Rica: los contenidos de los textos de la editorial incluyen las adecuaciones para que sirvan al propósito educativo de la UNED.

7. Vale agregar que en la UNED la producción de cursos en línea por parte de las cátedras ha generado poco a poco la consciencia de producción flexible de módulos -en soporte digital- tanto para efectos de la adecuación de contenidos de textos-expertos, como para la actualización de guías de estudio, siguiendo las demandas curriculares. Esto representa una vía distinta de la que llevaría -compulsivamente- a la producción de guías impresas en soporte papel, ya que el soporte digital permite actualizaciones de más bajos costos y más rápida intervención. La principal dificultad está relacionada con la cobertura de equipo informático para los estudiantes. Cabría revisar críticamente, a la luz de las nuevas tecnologías de la información y comunicación, qué aspectos de una guía de estudio de tipo impreso pierden vigencia dentro del esquema de producción y diseño digital. Este aspecto de suyo tan importantísimo, va a ser tangencialmente atendido en este artículo, por razones de tiempo y espacio.

8. Tengamos en mente -a guisa de ejemplo- la importancia de una perspectiva crítica ante la debacle económica que la administración financiera estadounidense ha generado a raíz de las hipotecas; la crisis de recesión económica de todo el sistema capitalista actual, ha llevado graves consecuencias en algunos ámbitos como el del empleo. Sensatamente alguien podría demandar teorías de la Administración que tomen nota crítica de estas circunstancias. Por ejemplo, en el enfoque del Dr. Rodríguez Matos, invitado por la UNED, se presentaron teorías con perspectiva crítica para una administración de carácter latinoamericano que prevén con criterio nacionalista los intereses financieros internacionales. Las conferencias ofrecidas por este especialista están contenidas en su obra: Rodríguez, M. (2005) La estructura de la administración crítica (Una interpretación dialéctica). Asociación Puertorriqueña de Profesores Universitarios. San Juan. Puerto Rico. 205 P.

9. Un ejemplo de esto lo tendríamos con la Escuela de Administración que ha desarrollado convenio
(UNED-Pearson) mediante el cual se le introduce a los libros de esta firma aspectos didácticos solicitados por la cátedra correspondiente. Esta modalidad prácticamente prescinde de los servicios de la instancia de producción de materiales didáctico, que a lo sumo desempeña una función de supervisión de calidad de lo que la cátedra solicitó a la editorial.

10. El profesor Eugenio Echeverría (desde la Filosofía para Niños: FpN) define como característica del programa de enseñanza filosófica aquella que "parte del convencimiento de que si alguien entiende y sabe algo, debe ser capaz de explicarlo de manera que la mayoría de las personas también lo comprendan". Filosofía para Niños. (2004) SM Ediciones. Madrid. España. Este postulado de "claridad" en FpN puede llevar a interpretar el trabajo didáctico como tarea de traducción. No obstante, existe una relatividad inherente al concepto de "claridad" en pedagogía puesto que está sujeto directamente a las intencionalidades de comunicación según los destinatarios de los mensajes educativos de la enseñanza: D'Alton: (1994) El análisis del discurso y los materiales didácticos. EUNED. San José, Costa Rica. Esta autora "aclara" que el lenguaje es -de por sí- "abierto" a distintos significados, y que no existe manera de sujetarlo mono semánticamente y esta condición de "apertura" que le es propia, es, per se, luz y sombra de nuevos sentidos. La explicación estriba en el carácter de ambigüedad como rasgo intrínseco del lenguaje y con el cual se las tiene que ver la didáctica.

11. En este sentido disentimos radicalmente de aquellas posiciones investigativas que realizan consultas a los estudiantes respecto de los contenidos curriculares como si éstos fueran responsabilidad de ellos y no de la institución educativa. Lamentablemente, la didáctica que se desprende de semejante delegación, atenta contra los estándares académicos básicos de la Educación Superior.

12. Ver: D'Alton C. (1994) El análisis del discurso y los materiales didácticos. EUNED. San José, Costa Rica. También, ver: Segura L. (1993) La dimensión comunicativa en la producción de materiales impresos para la educación a distancia. Revista Iberoamericana de Educación Superior a Distancia. Vol. 5, No 3 (Junio, 1993). A.I.E.S.A.D Madrid, España.

\section{REFERENCIAS}

Assmann, H. (2002). Placer y ternura en la educación. Ediciones Narcea. Madrid. España.

Barthes, R. (1971, 59-89). Elementos de semiología. Ediciones Comunicación. Madrid. España. 
Bernstein, B. (2000). Hacia una sociología del discurso pedagógico. Ediciones Magisterio. Bogotá. Colombia.

Bohm, D. (1987). La totalidad y el orden implicado. Editorial Kayros. Barcelona. España.

Camacho, L. (1978). Lógica dialéctica y lógica formal: hacia una precisión mayor en los términos, conceptos y métodos. Revista de Filosofía de la Universidad de Costa Rica, XVI (44), 150-157. Universidad de Costa Rica. San José. Costa Rica.

Castillo, Th. (2008). Recorrido histórico acerca de la calidad y producción de los materiales didácticos: problemas identificados del 2005 al 2008. Sistematización de talleres de la Vicerrectoría Académica. UNED. San José. Costa Rica.

Chadwick, C. (1999). El uso de los principios de diseño de enseñanza aprendizaje en la prepación de textos escolares. Umbral 2000. Barcelona. España. Consultado el 15 de setiembre de 2010, http://white.oit.org.pe/spanish/26oameri/oitre g/activid/proyectos/actrav/edob/material/pdf/ archiv024.pdf.

Chevallard, Y. (2000). Transposición Didáctica: Del saber sabio al saber enseñado. Ediciones AlQUÉ. Buenos Aires. Argentina

Claramount, M.C. (2006). Casitas quebradas: El problema de la violencia doméstica en Costa Rica. Décima reimpresión. EUNED. San José, Costa Rica.

D'Alton, C. (1994). El análisis del discurso y los materiales didácticos. EUNED. San José. Costa Rica.

De Faria, C. E. (2006, 79-104). Ingeniería didáctica. Cuadernos de investigación y formación en educación matemática. Centro de Investigaciones Matemáticas y Meta-matemáticas. Universidad de Costa Rica. San José. Costa Rica.

DELPHI: Técnica de consulta a expertos. Consultado el 15 de Junio de 2010, http://www.gtic.ssr. upm.es/encuestas/delphi.htm.

Dondis, D. (1992). La sintaxis de la imagen. Ediciones Gustavo Gili Gaya. Madrid. España.

Echeverría, E. (2004). Filosofía para Niños. SM Ediciones. Madrid. España.
Galichet, F. (2000) La didáctica de la filosofía en Francia: debates y perspectivas. Ediciones Novedades Educativas. Buenos Aires. Argentina.

García Aretio, L. Componentes básicos de un curso a distancia. Iberoamericana de Educación Superior a Distancia, Vol. 5, No 3, 68-90. Madrid. España.

Guiraud, P. (1983) La semiología. Ediciones Siglo XXI. Madrid. España.

Gutiérrez, F. y Prieto D. (2004). Mediación Pedagógica. Ediciones La Copia Fiel. Guatemala. Guatemala.

Huffman, D. (2009, Febrero). Seminario Taller Métodos y metódica científica. SEP-Universidad Estatal a Distancia (Sede Central Sabanilla, San Pedro de Montes de Oca). San José. Costa Rica.

Informe Delors. (1998). Pilares de la educación mundial. Consultado el 15 de Setiembre de 2010, http://www.unesco.org/education/pdf/ DELORS_S.PDF

Jorba y Casellas (Editores). (1997). Estrategias y técnicas para la gestión social en el aula. Volumen I: La regulación y la autorregulación de los aprendizajes. Editorial SÍNTESIS. Barcelona, España.

Jornada de encuentro de Física: Entre la UCR y la Universidad de Jaén (27-29 de Febrero, 2009). Universidad de Costa Rica (Sede Rodrigo Facio). San José. Costa Rica.

Mandrioni, H. (1964, 103-126). Introducción a la filosofía. Editorial Kapelusz. Madrid. España.

Manual de estilos del diario argentino La Nación. Consultado el 15 de Setiembre de 2010, http:// www.educared.org.ar/periodismo/curso/ clase\%207/pe_07_02.htm

Núñez, D. (2002). Derechos humanos y transversalidad curricular: Una propuesta para América Latina. Revista Espiga. No 5, p. 63-80. Universidad Estatal a Distancia. San José. Costa Rica.

Núñez, D. (1999). Transformar o repetir conocimientos: experiencia con materiales didácticos. Revista Innovaciones Educativas. № 10, p.15-27. Universidad Estatal a Distancia. San José. Costa Rica. 
Núñez, D. (2009). Lineamientos y criterios sobre Guías de estudio para libros de mercado, en UNED-Costa Rica. Informe Final de Investigación. Vicerrectoría de Investigación. Universidad Estatal a Distancia. San José. Costa Rica.

Núñez, D. (2002). UNED virtual y periodismo electrónico: insumos para debatir. Revista Innovaciones Educativas. No 13, p.55-66. Universidad Estatal a Distancia. San José. Costa Rica.

Núñez, D. (2004). Filosofía para niños y sistema de Educación a Distancia. Revista Innovaciones Educativas. No 14, p. 81-92. Universidad Estatal a Distancia. San José. Costa Rica.

Núñez, D. (2009.) Validación de materiales didácticos: el caso de la guía de estudio. Ponencia en el Programa de Producción de Materiales Impresos -PROMAI-Febrero, 2009.

Prieto, D. (1982). Diseño y Comunicación. Universidad Autónoma Metropolitana. México. México.

PROIFED. (2008). Documento de trabajo: Los modelos pedagógicos centrados en el estudiante: apuntes sobre los procesos de aprendizaje y enseñanza (noviembre, 2008). Vicerrectoría de Investigación. Universidad Estatal a Distancia. San José. Costa Rica.

Ramírez, E. A. (2003). Semiótica y textos didácticos. Análisis de una práctica textual. Editorial Kimpres. Bogotá. Colombia.

Rodríguez, M. (2005) La estructura de la administración crítica (Una interpretación dialéctica). Asociación Puertorriqueña de Profesores Universitarios. San Juan. Puerto Rico.

Segura, L. M. (1993,43-60). La dimensión comunicativa en la producción de materiales impresos para la educación a distancia. Revista Iberoamericana de Educación superior a Distancia. Vol. V-No3, Junio 1993. AIESAD. Madrid. España.

UNED. (2009) Directrices de la UNED. CIDREB. San José. Costa Rica. 